\title{
Delay Analysis of Coupled Transmission Lines *
}

\author{
Andrew B. Kahng \\ UCLA Computer Science Department \\ Los Angeles, CA 90095-1596 USA \\ abk@cs.ucla.edu
}

\author{
Sudhakar Muddu \\ Silicon Graphics, Inc. \\ Mountain View, CA 94039 USA \\ muddu@mti.sgi.com
}

\begin{abstract}
In this paper, we analyze coupled transmission lines and obtain a relationship between the moments of the coupled transfer functions. We then derive expressions for the first and second moments of the coupled transfer function which can be used to compute the response and threshold delays under various input excitations. We can compute the interconnect delay under parasitic coupling effects by using the analytic delay formulas given in $[8,9]$ for step and ramp inputs. We also present the analysis to compute the general $k^{\text {th }}$ moment for the coupled interconnect lines.
\end{abstract}

\section{Introduction}

With the rapid evolution of VLSI technology, minimum feature size and the distance between interconnections continue to decrease. Thus, transmission line effects such as crosstalk will play an important role in determining system performance. Crosstalk effects in voltage response are due to mutual capacitances and inductances between adjacent lines. The coupling between lines increases as the separation between the lines decreases, as the distance to the ground plane increases, and as the distance over which the lines neighbor each other increases. In addition, crosstalk effects increase as characteristic impedance $\left(Z_{0}\right)$ increases since capacitance of the line to the ground plane decreases and the coupling capacitance will have greater effect on the signal voltage [1]. Numerical methods accurately calculate interconnect capacitances using finite element methods $[3,13]$, but are too time consuming for performance-driven layout synthesis. Thus, approximate analytical formulas for parallel plate, fringe, and (lateral) coupling capacitances have been proposed by Sakurai et al. [15], Meijs et al. [16] and others. Similarly, Sakurai et al. [15] have proposed an analytical formula for the coupling capacitance between two lines as

$\Lambda=\left[0.03 \varepsilon_{o x}\left(\frac{w}{h}\right)+0.83 \varepsilon_{o x}\left(\frac{t}{h}\right)-0.07 \varepsilon_{o x}\left(\frac{t}{h}\right)^{0.222}\right]\left(\frac{s}{h}\right)^{-1.34}$

where $w$ is the width of the line, $t$ is the thickness of the line, $s$ is the space between the two lines, $h$ is the height of the line above the ground plane, and $l$ is the length of the line. However, such formulas do not address the estimation of delay due to noise coupling.

Crosstalk noise in coupled lines can either increase or decrease signal propagation delays. This depends on the line impedances and on whether the neighboring lines are driven by the same input voltage or different input voltages (for example, one input rising and the other input falling) or no input voltage (i.e., line is quiet). Many works deal with the analysis, design, and applications of coupled microstriplines and transmission lines. Initial analyses of crosstalk due to capacitive charge sharing between lossless transmission lines was given in $[5,2]$. The analysis was based on odd and even modes of propagation with separate impedances and propagation speeds, and the voltage response of the signal traveling on the coupled transmission lines was expressed as a superposition of these two modes. Reviews of this method are given in [1]. The effects of crosstalk in multiconductor lossy transmission lines were analyzed using Fourier transform techniques and numerical methods in [17]. Recently, Sriram and Kang [14] proposed a technique which recursively com-

\footnotetext{
*This work was supported by NSF Young Investigator Award MIP-9257982. ABK is currently Visiting Scientist at Cadence Design Systems, Inc. (on sabbatical leave from UCLA), email: ak@ cadence.com.
}

putes the admittance and coefficients of the coupled transfer functions. All these simulation techniques can be used to calculate the transient response, but are less applicable to the synthesis of the interconnection topology and layout. Basically, with these methods the simulation step becomes a bottleneck in the design process (e.g., consider multiple nets in a high-density MCM substrate).

In this paper, we analyze the crosstalk between on-chip interconnections by modeling each line by an infinite number of $R L C$ segments. Coupling between lines is similarly modeled as an infinite number of coupled capacitances between pairs of corresponding nodes of each line. We give a relationship between the coefficients of the coupled transfer functions; this reduces the computation effort for all transfer function coefficients. We then derive expressions for the first and second moments of the transfer function, which will be used to compute threshold delay under various input excitations. Then, analytic delay formulas based on the first few moments can be applied for step input [8] and for ramp input [9]. We also discuss crosstalk response computation for a coupled system. The analysis can be extended to non-identical coupled lines (e.g., each line having different width, etc.) to derive first and second moments of the coupled transfer functions.

\section{Analysis of a Single Lossy Transmission Line}

Various techniques $[10,12]$ have been proposed for the simulation of interconnects modeled as a single lossy transmission line. SPICE gives the most accurate insight into arbitrary interconnect structures, but is expensive. Moment based approaches [11, 14] model the individual interconnects using a large number of uniform $R L C$ segments; the accuracy of these methods can be close to SPICE, but the time complexity is too high for iterative layout optimization. Faster techniques such as the two-pole approach $[18,7]$ have been used to calculate the response to reasonable accuracy using the first and second moments, and $[8,9]$ developed analytical (closed-form) delay models for both step and ramp input based on the first few moments of the transfer function. We now briefly review the computation of transfer function moments (or coefficients), which is basic to the analysis of coupled transmission lines.

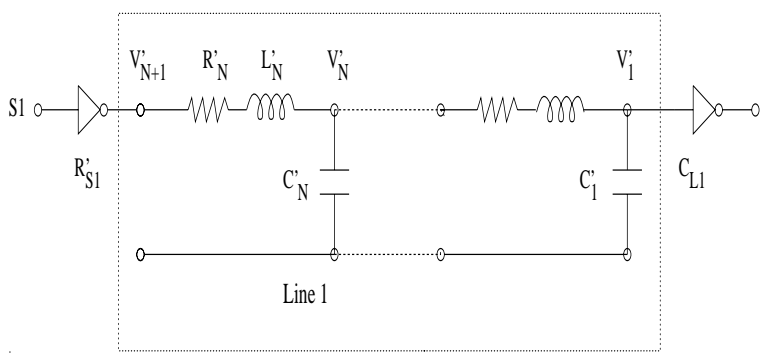

Figure 1: An $N$-segment RLC model for Line 1 in a system of coupled lines.

The general equivalent circuit with $N R L C$ segments (Figure 1) can be used to model a distributed $R L C$ line. For Line 1 in a system of coupled lines, source resistance (i.e., the driving gate resistance) is represented by $R_{S 1}^{\prime}$ and the load capacitance (i.e., target gate input capacitance) is $C_{L 1}$. The distributed line parameters, such as the moments, can be obtained by setting the number of segments $N \rightarrow \infty$. 
From nodal equations, the voltage $V_{S 1}^{\prime}$ at the source node is

$$
\begin{aligned}
V_{S 1}^{\prime}(s)= & {\left[V_{N}^{\prime}(s)+R_{S 1}^{\prime}\left(\sum_{j=1}^{N} s C_{j}^{\prime} V_{j}^{\prime}(s)+s C_{L 1} V_{1}^{\prime}(s)\right)\right.} \\
& \left.+\left(R_{N}^{\prime}+s L_{N}^{\prime}\right) \sum_{j=1}^{N} s C_{j}^{\prime} V_{j}^{\prime}(s)+\left(R_{N}^{\prime}+s L_{N}^{\prime}\right) s C_{L 1} V_{1}^{\prime}(s)\right] \\
= & {\left[V_{1}^{\prime}(s)+\left(\sum_{j=1}^{N} s C_{j}^{\prime} V_{j}^{\prime}(s)+s C_{L 1} V_{1}^{\prime}(s)\right) R_{S 1}^{\prime}\right.} \\
& \left.+\sum_{j=1}^{N} s C_{j}^{\prime} V_{j}^{\prime}(s) \sum_{i=j}^{N} R_{i}^{\prime}+s L_{i}^{\prime}+s C_{L 1} V_{1}^{\prime}(s) \sum_{i=1}^{N} R_{i}^{\prime}+s L_{i}^{\prime}\right]
\end{aligned}
$$

where $V_{1}^{\prime}(s)$ is the voltage at the load of Line 1. Without loss of generality, we represent the ratio of node voltage $V_{i}^{\prime}$ to the load voltage $V_{1}^{\prime}$ as a series in $s$ :

$$
\frac{V_{i}^{\prime}(s)}{V_{1}^{\prime}(s)}=b_{0}^{i}+b_{1}^{i} s+b_{2}^{i} s^{2}+b_{3}^{i} s^{3}+\ldots=H_{1}^{S L}(s)
$$

where $b_{0}^{i}=1$ and $H_{1}^{S L}(s)$ represents the reciprocal of the Line 1 transfer function. In general, the $k^{\text {th }}$ coefficient of the transfer function can be obtained by using the recursive equation [7]

$$
b_{k}^{N+1}=R_{N}^{\prime} \sum_{j=1}^{N} C_{j}^{\prime} \cdot b_{k-1}^{j}+L_{N}^{\prime} \sum_{j=1}^{N} C_{j}^{\prime} \cdot b_{k-2}^{j}+b_{k}^{N}
$$

The exact values of the coefficients for the distributed line are obtained by substituting $R_{j}^{\prime}=\frac{R^{\prime}}{N}, L_{j}^{\prime}=\frac{L^{\prime}}{N}$ and $C_{j}^{\prime}=\frac{C^{\prime}}{N}$ and setting $N \rightarrow \infty$ [7], where $R^{\prime}, C^{\prime}$, and $L^{\prime}$ are the total resistance, capacitance and inductance of Line 1 :

$$
\begin{aligned}
b_{1}^{S L}= & R_{S 1}^{\prime}\left(C^{\prime}+C_{L 1}\right)+R^{\prime}\left(\frac{C^{\prime}}{2}+C_{L 1}\right) \\
b_{2}^{S L}= & \frac{R_{S 1}^{\prime} R^{\prime} C^{\prime 2}}{6}+\frac{R_{S 1}^{\prime} R^{\prime} C^{\prime} C_{L 1}}{2}+\frac{\left(R^{\prime} C^{\prime}\right)^{2}}{24}+\frac{R^{\prime 2} C^{\prime} C_{L 1}}{6} \\
& +L^{\prime}\left(\frac{C^{\prime}}{2}+C_{L 1}\right)
\end{aligned}
$$

The Elmore delay for Line $\mathbf{1}$ is given by the transfer function coefficient $b_{1}$. The transfer function and Elmore delay for Line 2 alone are expressed similarly, e.g., $H_{2}^{S L}(s)$ denotes the reciprocal of the uncoupled Line 2 transfer function.

\section{Analysis of Lossy Coupled Transmission Lines}

We now compute the voltage response and the transfer function for two coupled interconnects, modeled as shown in Figure 2. We also derive new Elmore delay expressions for the coupled lines under various cases of input excitation: (i) one line is active (rising/falling input) and the other line is quiet, and (ii) the two lines are driven by opposite inputs.

We write the interconnect parameters for Line 1 as $R_{j}^{\prime}, C_{j}^{\prime}$ and $L_{j}^{\prime}$ and for Line 2 as $R_{j}^{\prime \prime}, C_{j}^{\prime \prime}$ and $L_{j}^{\prime \prime}$. Let $R_{S 1}^{\prime}$ and $R_{S 2}^{\prime \prime}$ be the respective source resistances, and let $C_{L 1}$ and $C_{L 2}$ be the respective load capacitances. The voltages at node $j$ of Line 1 and of Line 2 are $V_{j}^{\prime}(s)$ and $V_{j}^{\prime \prime}(s)$ respectively. ${ }^{1}$ Since the interconnects are distributed, the coupling capacitance (or mutual capacitance) is also distributed in nature; we model it by $N$ capacitances $\left(\Lambda_{j}\right)$ between corresponding node segments of the two lines.

${ }^{1}$ In general, parameters marked with ' refer to Line 1, while those marked with " refer to Line 2 .

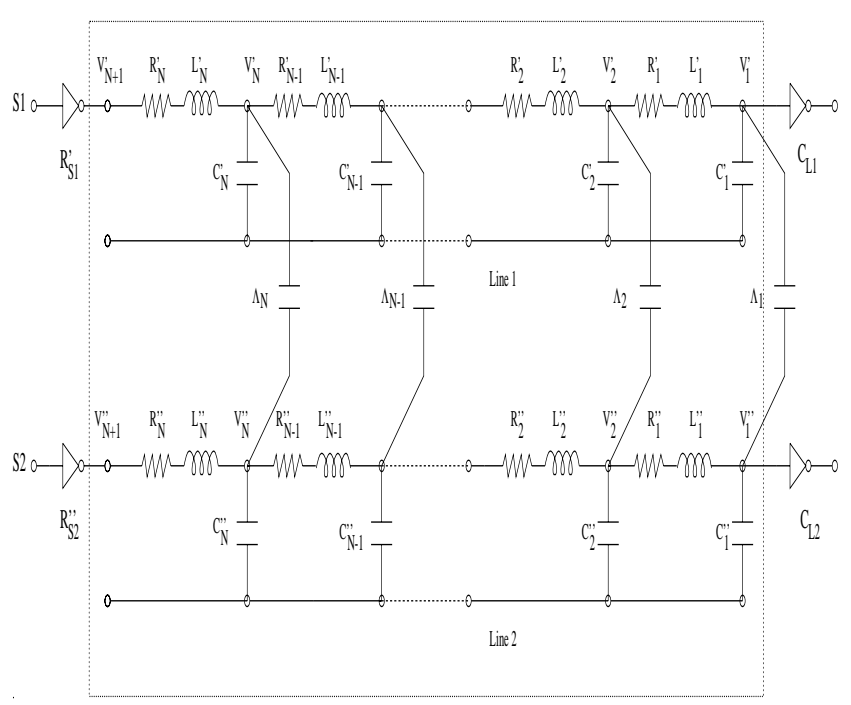

Figure 2: $N$-segment RLC equivalent circuits for two coupled transmission lines.

Extending the analysis of the previous section, the voltage at the source node of Line 1 can be expressed using the recursive equation

$$
\begin{aligned}
V_{S 1}^{\prime}(s)= & V_{N}^{\prime}(s)+R_{S 1}^{\prime}\left(\sum_{j=1}^{N} s C_{j}^{\prime} V_{j}^{\prime}(s)+\sum_{j=1}^{N} s \Lambda_{j}\left(V_{j}^{\prime}(s)-V_{j}^{\prime \prime}(s)\right)\right. \\
& \left.+s C_{L 1} V_{1}^{\prime}(s)\right)+\left(R_{N}^{\prime}+s L_{N}^{\prime}\right)\left(\sum_{j=1}^{N} s C_{j}^{\prime} V_{j}^{\prime}(s)\right. \\
& \left.+\sum_{j=1}^{N} s \Lambda_{j}\left(V_{j}^{\prime}(s)-V_{j}^{\prime \prime}(s)\right)\right)+\left(R_{N}^{\prime}+s L_{N}^{\prime}\right) s C_{L 1} V_{1}^{\prime}(s) \\
= & R_{S 1}^{\prime} \sum_{j=1}^{N} s\left(C_{j}^{\prime}+\Lambda_{j}\right) V_{j}^{\prime}(s)+\sum_{j=1}^{N} s\left(C_{j}^{\prime}+\Lambda_{j}\right) V_{j}^{\prime}(s) \sum_{i=j}^{N} R_{i}^{\prime}+s L_{i}^{\prime} \\
& +s C_{L 1}\left(R_{S 1}^{\prime}+\sum_{i=1}^{N}\left(R_{i}^{\prime}+s L_{i}^{\prime}\right)\right) V_{1}^{\prime}(s)+V_{1}^{\prime}(s) \\
& -R_{S 1}^{\prime} \sum_{j=1}^{N} s \Lambda_{j} V_{j}^{\prime \prime}(s)-\sum_{j=1}^{N} s \Lambda_{j} V_{j}^{\prime \prime}(s) \sum_{i=j}^{N}\left(R_{i}^{\prime}+s L_{i}^{\prime}\right)
\end{aligned}
$$

Recall from Equation (1) that the ratio of node voltage to target load voltage can be expressed as an infinite series. For Line 1, we write $\frac{V_{i}^{\prime}(s)}{V_{1}^{\prime}(s)}=b_{0}^{i}+b_{1}^{i} s+b_{2}^{i} s^{2}+b_{3}^{i} s^{3}+\ldots=\sum_{k=0}^{\infty} b_{k}^{i} s^{k}$ and for Line 2 we write $\frac{V_{i}^{\prime \prime}(s)}{V_{1}^{\prime \prime}(s)}=B_{0}^{i}+B_{1}^{i} s+B_{2}^{i} s^{2}+B_{3}^{i} s^{3}+\ldots=\sum_{k=0}^{\infty} B_{k}^{i} s^{k}$. Expressing the node voltages in Equation (4) in terms of these infinite series and the target load voltages, we have

$$
\begin{aligned}
V_{S 1}^{\prime}(s)= & V_{1}^{\prime}(s)\left[1+\left(R_{S 1}^{\prime}+\sum_{i=1}^{N}\left(R_{i}^{\prime}+s L_{i}^{\prime}\right)\right) s C_{L 1}\right. \\
& \left.+\sum_{j=1}^{N} s\left(C_{j}^{\prime}+\Lambda_{j}\right)\left(\sum_{k=0}^{\infty} b_{k}^{j} s^{k}\right)\left(R_{S 1}^{\prime}+\sum_{i=j}^{N}\left(R_{i}^{\prime}+s L_{i}^{\prime}\right)\right)\right] \\
& -V_{1}^{\prime \prime}(s)\left[\sum_{j=1}^{N} s \Lambda_{j}\left(\sum_{k=0}^{\infty} B_{k}^{j} s^{k}\right)\left(R_{S 1}^{\prime}+\sum_{i=j}^{N}\left(R_{i}^{\prime}+s L_{i}^{\prime}\right)\right)\right] \\
= & H_{11}(s) V_{1}^{\prime}(s)+H_{12}(s) V_{1}^{\prime \prime}(s)
\end{aligned}
$$

Similarly, the voltage at the source of Line 2 is

$$
V_{S 2}^{\prime \prime}(s)=V_{1}^{\prime \prime}(s)\left[1+\left(R_{S 2}^{\prime \prime}+\sum_{i=1}^{N}\left(R_{i}^{\prime \prime}+s L_{i}^{\prime \prime}\right)\right) s C_{L 1}\right.
$$




$$
\begin{aligned}
& \left.+\sum_{j=1}^{N} s\left(C_{j}^{\prime \prime}+\Lambda_{j}\right)\left(\sum_{k=0}^{\infty} B_{k}^{j} s^{k}\right)\left(R_{S 2}^{\prime \prime}+\sum_{i=j}^{N}\left(R_{i}^{\prime \prime}+s L_{i}^{\prime \prime}\right)\right)\right] \\
& -V_{1}^{\prime}(s)\left[\sum_{j=1}^{N} s \Lambda_{j}\left(\sum_{k=0}^{\infty} b_{k}^{j} s^{k}\right)\left(R_{S 2}^{\prime \prime}+\sum_{i=j}^{N}\left(R_{i}^{\prime \prime}+s L_{i}^{\prime \prime}\right)\right)\right] \\
& =H_{21}(s) V_{1}^{\prime}(s)+H_{22}(s) V_{1}^{\prime \prime}(s)
\end{aligned}
$$

Observe that the voltage on Line 1 depends on both sets of line parameters, the coupling capacitance, and both target voltages. The polynomial $H_{11}(s)$ represents the reciprocal of the transfer function of Line 1 with $(C+\Lambda)$ as the total line capacitance. Note that $H_{11}(s)$ can be obtained by replacing the capacitance terms $C_{j}^{\prime}$ by $\left(C_{j}^{\prime}+\Lambda_{j}\right)$ terms in the uncoupled Line 1 transfer function $H_{1}^{S L}(s)$. However, the polynomial $\mathrm{H}_{12}(s)$, which represents the coupled transfer function between the lines, is different from the uncoupled polynomial $H_{2}^{S L}(s)$ of Line 2.

Equations (5) and (6) relate the source node voltages to the target load voltages as

$$
\left(\begin{array}{c}
V_{S 1}^{\prime}(s) \\
V_{S 2}^{\prime \prime}(s)
\end{array}\right)=\left(\begin{array}{ll}
H_{11}(s) & H_{12}(s) \\
H_{21}(s) & H_{22}(s)
\end{array}\right)\left(\begin{array}{c}
V_{1}^{\prime}(s) \\
V_{1}^{\prime \prime}(s)
\end{array}\right)
$$

and inverting this equation allows us to express the load voltages in terms of source voltages as

$$
\left(\begin{array}{c}
V_{1}^{\prime}(s) \\
V_{1}^{\prime \prime}(s)
\end{array}\right)=\frac{1}{|H|}\left(\begin{array}{cc}
H_{22}(s) & -H_{12}(s) \\
-H_{21}(s) & H_{11}(s)
\end{array}\right)\left(\begin{array}{c}
V_{S 1}^{\prime}(s) \\
V_{S 2}^{\prime \prime}(s)
\end{array}\right)
$$

where $|H|=\left(H_{11}(s) H_{22}(s)-H_{12}(s) H_{21}(s)\right)$ denotes the matrix determinant.

\section{Relationship Between Coupled Polynomials}

The source voltages and the target load voltages of the coupled lines are related through the four polynomials given by Equation (7). We now derive relationships between the coefficients of the coupled polynomials. Consider the case when Line 1 and Line 2 have identical parameters, i.e.

$$
R_{j}^{\prime}=R_{j}^{\prime \prime}=\frac{R}{N}, \quad C_{j}^{\prime}=C_{j}^{\prime \prime}=\frac{C}{N}, \quad L_{j}^{\prime}=L_{j}^{\prime \prime}=\frac{L}{N}, \quad \Lambda_{j}=\frac{\Lambda}{N}
$$

where $R, L$ and $C$ are the total resistance, inductance and capacitance of each line and $\Lambda$ is the total coupling capacitance between the two lines. The coupled polynomial coefficients and the first moment of the corresponding transfer function can be obtained by substituting these parameters into Equations (5) and (6) and letting $N \rightarrow \infty$. The coupled polynomial $H_{11}(s)$ is

$$
\begin{aligned}
H_{11}(s)= & \lim _{N \rightarrow \infty}\left[1+\left(R_{S 1}^{\prime}+\sum_{i=1}^{N}\left(R_{i}^{\prime}+s L_{i}^{\prime}\right)\right) s C_{L 1}\right. \\
& \left.+\sum_{j=1}^{N} s\left(C_{j}^{\prime}+\Lambda_{j}\right)\left(\sum_{k=0}^{\infty} b_{k}^{j} s^{k}\right)\left(\sum_{i=j}^{N}\left(R_{i}^{\prime}+s L_{i}^{\prime}\right)+R_{S 1}^{\prime}\right)\right] \\
= & 1+b_{1}^{11} s+b_{2}^{11} s^{2}+\ldots+b_{k}^{11} s^{k}+\ldots
\end{aligned}
$$

Collecting all terms with coefficients of $s$ and $s^{2}$, we get

$$
\begin{aligned}
b_{1}^{11}= & R_{S 1}^{\prime}\left(C+\Lambda+C_{L 1}\right)+R\left(\frac{(C+\Lambda)}{2}+C_{L 1}\right) \\
b_{2}^{11}= & \lim _{N \rightarrow \infty} R_{S 1}^{\prime} \frac{(C+\Lambda)}{N} \sum_{j=1}^{N} b_{1}^{j}+\frac{R(C+\Lambda)}{N^{2}} \sum_{j=1}^{N}(N-j+1) b_{1}^{j} \\
& +\frac{L(C+\Lambda)}{N^{2}} \sum_{j=1}^{N}(N-j+1) \\
= & \frac{R_{S 1}^{\prime} R(C+\Lambda)^{2}}{6}+\frac{R_{S 1}^{\prime} R(C+\Lambda) C_{L 1}}{2}+\frac{(R(C+\Lambda))^{2}}{24} \\
& +\frac{R^{2}(C+\Lambda) C_{L 1}}{6}+L^{\prime}\left(\frac{(C+\Lambda)}{2}+C_{L 1}\right)
\end{aligned}
$$

The coupled polynomial $H_{12}(s)$ can be expanded as

$$
\begin{aligned}
H_{12}(s)= & \lim _{N \rightarrow \infty}\left[-\sum_{j=1}^{N} s \Lambda_{j}\left(\sum_{k=0}^{\infty} B_{k}^{j} s^{k}\right)\left(\sum_{i=j}^{N}\left(R_{i}^{\prime}+s L_{i}^{\prime}\right)+R_{S 1}^{\prime}\right)\right] \\
= & \lim _{N \rightarrow \infty}\left[-\frac{s R_{S 1}^{\prime} \Lambda}{N}\left(\sum_{j=1}^{N} \sum_{k=0}^{\infty} B_{K}^{j} s^{k}\right)\right. \\
& \left.-\frac{(R+s L) s \Lambda}{N^{2}} \sum_{j=1}^{N}(N-j+1)\left(\sum_{k=0}^{\infty} B_{k}^{j} s^{k}\right)\right] \\
= & -\left[b_{1}^{12} s+b_{2}^{12} s^{2}+\ldots\right]
\end{aligned}
$$

Similarly, the polynomials $H_{21}(s)$ and $H_{22}(s)$ can be expanded as infinite series. Notice that the polynomials $H_{11}(s)$ and $H_{21}(s)$ have many terms in common, as do $H_{22}(s)$ and $H_{12}(s)$. The relationship between the coefficients of polynomials $H_{11}(s)$ and $H_{21}(s)$ are

$$
\begin{aligned}
b_{1}^{21}= & \frac{-\Lambda}{(C+\Lambda)}\left(b_{1}^{11}-R_{S 1}^{\prime} C_{L 1}-R C_{L 1}\right)+\left(R_{S 1}^{\prime}-R_{S 2}^{\prime \prime}\right) \Lambda \\
b_{2}^{21}= & \frac{-\Lambda\left(b_{2}^{11}-L C_{L 1}\right)}{(C+\Lambda)}+\frac{\left(R_{S 1}^{\prime}-R_{S 2}^{\prime \prime}\right) R(C+\Lambda) \Lambda}{6} \\
& +\frac{\left(R_{S 1}^{\prime}-R_{S 2}^{\prime \prime}\right) R \Lambda C_{L 1}}{2} \\
b_{k}^{21}= & \lim _{N \rightarrow \infty} \frac{-\Lambda}{(C+\Lambda)} \cdot b_{k}^{11}+\frac{\left(R_{S 1}^{\prime}-R_{S 2}^{\prime \prime}\right) \Lambda}{N} \sum_{j=1}^{N} b_{k-1}^{j} \text { for } k \geq 3
\end{aligned}
$$

The coefficients of polynomials $H_{22}(s)$ and $H_{12}(s)$ are related as

$$
\begin{aligned}
b_{1}^{12}= & \frac{-\Lambda}{(C+\Lambda)}\left(b_{1}^{22}-R_{S 2}^{\prime \prime} C_{L 2}-R C_{L 2}\right)+\left(R_{S 2}^{\prime \prime}-R_{S 1}^{\prime}\right) \Lambda \\
b_{2}^{12}= & \frac{-\Lambda\left(b_{2}^{22}-L C_{L 2}\right)}{(C+\Lambda)}+\frac{\left(R_{S 2}^{\prime \prime}-R_{S 1}^{\prime}\right) R(C+\Lambda) \Lambda}{6} \\
& +\frac{\left(R_{S 2}^{\prime \prime}-R_{S 1}^{\prime}\right) R \Lambda C_{L 2}}{2} \\
b_{k}^{12}= & \lim _{N \rightarrow \infty} \frac{-\Lambda}{(C+\Lambda)} \cdot b_{k}^{22}+\left(R_{S 2}^{\prime \prime}-R_{S 1}^{\prime}\right) \Lambda\left(\sum_{j=1}^{N} B_{k-1}^{j}\right) \text { for } k \geq 3
\end{aligned}
$$

These relationships between the coefficients of the coupled polynomials can be used to efficiently compute the moments and response for coupled lines.

\section{Configurations of Coupled Lines}

We now analyze the coupled interconnect lines to compute the moments of the coupled system, and to estimate delays and crosstalk for three cases of input voltages at the source of the lines.

1. Line $\mathbf{1}$ has an active (rising or falling) source voltage while the Line 2 source is grounded (i.e., line is quiet). Using this analysis, a second case of the crosstalk on (quiet) Line $\mathbf{1}$ can be computed by applying a rising/falling source voltage to Line 2.

2. The two lines are driven by opposite inputs, i.e., Line $\mathbf{1}$ has a rising input and Line $\mathbf{2}$ has a falling input at the source.

\subsection{Active and Quiet Pair of Coupled Lines}

Consider a step input at the source of Line 1 and connect the source of Line 2 to ground as shown in Figure 3. For this configuration the transfer function can be computed by setting $V_{S 2}^{\prime \prime}(s)=0$ and $V_{S 1}^{\prime}(s)=$ $\frac{V_{0}}{s}$ in Equation (8):

$$
\frac{V_{1}^{\prime}(s)}{V_{S 1}^{\prime}(s)}=\frac{H_{22}(s)}{H_{11}(s) H_{22}(s)-H_{12}(s) H_{21}(s)}
$$




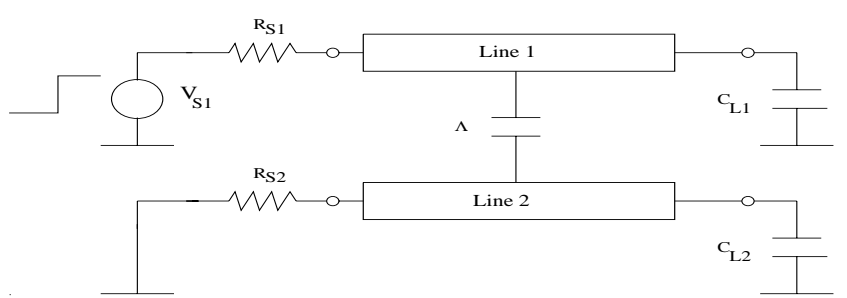

Figure 3: Two coupled lines with Line 1 being driven by a step inpu and Line 2 source being grounded.

Substituting for each polynomial with an infinite series,

$$
\begin{aligned}
\frac{V_{1}^{\prime}(s)}{V_{S 1}^{\prime}(s)} & =\frac{1+b_{1}^{22} s+b_{2}^{22} s^{2}+\ldots}{1+\left(b_{1}^{11}+b_{1}^{22}\right) s+\left(b_{2}^{11}+b_{2}^{22}+b_{1}^{11} b_{1}^{22}-b_{1}^{12} b_{1}^{21}\right) s^{2}+\ldots} \\
& =\frac{1+n_{1} s+n_{2} s^{2}+\ldots}{1+d_{1} s+d_{2} s^{2}+\ldots}
\end{aligned}
$$

The first moment (or Elmore delay) of the transfer function is obtained as

$$
M_{1}=d_{1}-n_{1}=R_{S 1}^{\prime}\left(C+\Lambda+C_{L 1}\right)+R\left(\frac{(C+\Lambda)}{2}+C_{L 1}\right)
$$

Clearly, the Elmore delay with the adjacent line being quiet is different from the Elmore delay of a single uncoupled line (Equation (3)): the difference between the two delays is that for the coupled line, the new capacitance is the sum of wire capacitance and all coupling capacitance. The second moment of the coupled system is

$$
M_{2}=\left(n_{2}-n_{1} d_{1}+d_{1}^{2}-d_{2}\right)=b_{1}^{11} b_{1}^{11}-b_{2}^{11}+b_{1}^{12} b_{1}^{21}
$$

Recently, it has been observed by many authors $[6,18,7,8,9]$ that a few (two or three) dominant poles of the transfer function are sufficient for accurate response computations with most present-day interconnect topologies. To compute threshold delays for the coupled interconnect system we can use the analytical delay formulas developed in [8] for step input (i.e., $V_{S 1}^{\prime}(s)=\frac{V_{0}}{s}$ ) and in [9] for ramp input (i.e., $V_{S 1}^{\prime}(s)=\frac{V_{0}}{T_{R} s^{2}}\left(1-e^{-s T_{R}}\right)$ ), where $T_{R}$ is the input ramp rise time.

The other case of an active and quiet pair of coupled lines estimates crosstalk noise at the end of Line 1 when the source of Line 1 is grounded and a step input is applied at the Line 2 source. In this case, the transfer function $\frac{V_{1}^{\prime}(s)}{V_{S 2}^{\prime \prime}(s)}$ is expressed in terms of the coupled polynomials and then approximated to the required accuracy.

\subsection{Active Coupled Lines with Opposite Inputs}

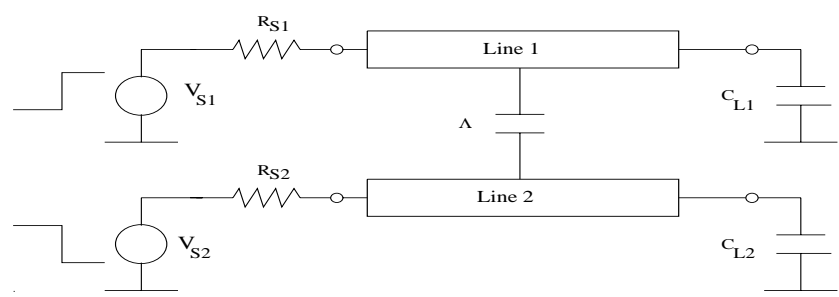

Figure 4: Two coupled lines with Line 1 being driven by a rising step input and Line 2 being driven by a falling step input.

Finally, we consider the case where Line 1 is driven by a rising step input and Line 2 is driven by a falling step input as shown in Figure 4 . The transfer function for this configuration can be calculated from Equation (8) by setting $V_{S 1}^{\prime}(s)=\frac{V_{0}}{s}$ and $V_{S 2}^{\prime \prime}(s)=-\frac{V_{0}}{s}=-V_{S 1}^{\prime}(s)$. The coupled transfer function is

$$
\frac{V_{1}^{\prime}(s)}{V_{S 1}^{\prime}(s)}=\frac{\left(H_{22}+H_{12}\right)}{H_{11}(s) H_{22}(s)-H_{12}(s) H_{21}(s)}
$$

$$
=\frac{\left.1+\left(b_{1}^{22}+b_{1}^{12}\right) s+\left(b_{2}^{22}+b_{2}^{12}\right) s^{2}+\ldots\right)}{1+\left(b_{1}^{11}+b_{1}^{22}\right) s+\left(b_{2}^{11}+b_{2}^{22}+b_{1}^{11} b_{1}^{22}-b_{1}^{12} b_{1}^{21}\right) s^{2}+\ldots}
$$

and the first (Elmore delay) and second moments are

$$
\begin{aligned}
& M_{1}=R_{S 1}^{\prime}\left(C+2 \Lambda+C_{L 1}\right)+R\left(\frac{C}{2}+\Lambda+C_{L 1}\right) \\
& M_{2}=b_{1}^{11}\left(b_{1}^{11}+b_{1}^{12}\right)+b_{1}^{12}\left(b_{1}^{22}+b_{1}^{21}\right)+b_{2}^{12}-b_{2}^{11}
\end{aligned}
$$

Again we can compute the threshold delays for the coupled system using the analytical delay formulas given in $[8,9]$.

\section{Conclusions}

We have presented the analysis of coupled transmission lines and the computation of moments for coupled transfer functions. We also describe a relationship between the moments of the coupled transfer functions, and derive expressions for the first and second moments of the transfer function which can be used to compute threshold delay under step input and ramp input.

\section{REFERENCES}

[1] H. B. Bakoglu, Circuits, Interconnectionsand Packagingfor VLSI, Addison Wesley, 1990.

[2] I. Catt, "Crosstalk (noise) in Digital Systems", IEEE Trans. Electronic Computers, vol. 16, Dec 1967, pp. 743-763.

[3] W. H. Chang, "Analytical IC Metal-Line Capacitance Formulas", IEEE Trans, Microwave Theory Tech. 9, Sep. 1976, pp. 608-611.

[4] W. C. Elmore, "The Transient Response of Damped Linear Networks with Particular Regard to Wideband Amplifiers", J. Applied Physics 19, Jan. 1948, pp. 55-63.

[5] A. Feller, H. R. Kaupp, and J. J. Digiacomo, "Crosstalk and Reflections in HighSpeed Digital Systems", Proc. Fall Joint Computer Conf., 1965, pp. 511-525.

[6] M. A. Horowitz, "Timing Models for MOS Circuits", PhD Thesis, Stanford University, Jan. 1984.

[7] A. B. Kahng and S. Muddu, "Efficient Analyses and Models of VLSI and MCM Interconnects", UCLA CS Dept. TR-950033, August 1995.

[8] A. B. Kahng and S. Muddu,' "Accurate Analytical Delay Models for VLSI Interconnects", IEEE Int. Symposium on Circuits and Systems, May 1996.

[9] A. B. Kahng, K. Masuko, and S. Muddu, "Analytical Delay Model for VLSI Interconnects Under Ramp Input”, To appear in IEEE ICCAD, Nov. 1996.

[10] L. T. Pillage and R. A. Rohrer, "Asymptotic Waveform Evaluation for Timing Analysis”, IEEE Trans. CAD, Apr. 1990, pp.352-366.

[11] C. L. Ratzlaff, N. Gopal, and L. T. Pillage, "RICS: Rapid Interconnect Circuit Evaluator", Proc. 28th ACM/IEEE Design Automation Conf., June 1991.

[12] J. S. Roychowdhuryand D. O. Pederson, "Efficient Transient Simulation of Lossy Interconnect", Proc. 28th ACM/IEEE Design Automation Conf., June 1991.

[13] A. E. Ruehli and P. A. Brennan, "Accurate Metallization Capacitances for Integrated Circuits and Packages", IEEE J. Solid-State Circuits 6, Aug. 1973, pp. 289-290.

[14] M. Sriram and S. M. Kang, "Fast Approximation of The Transient Response of Lossy Transmission Line Trees", Proc. ACM/IEEE Design Automation Conf., June 1993, pp. 691-696.

[15] T. Sakurai and K. Tamaru, "Simple Formulas for Two and Three Dimensional Capacitances", IEEE Trans. on Electron Devices 30, Feb. 1983.

[16] N. v. d. Meijs and J. T. Fokkema, "VLSI Circuit Reconstruction from Mask Topology", Integration 2, 1984, pp. 85-119.

[17] S. Voranantakul, J. L. Prince, and P. Hsu, "Crosstalk Analysis for High-Speed Pulse Propagation in Lossy Electrical Interconnections", IEEE Trans. Components, Hybrids, And Manufacturing Tech., Feb. 1993, pp. 127-136.

[18] D. Zhou, S. Su, F. Tsui, D. S. Gao and J. S. Cong, "A Simplified Synthesis of Transmission Lines with A Tree Structure", Intl. Journal of Analog Integrated Circuits and Signal Processing, Jan. 1994, pp. 19-30. 